nicht nur zu Hunger, sondern gerade zu Fleisch- und Fettnot."

Uber die Abweichungen vom Coulombsehen Gesetze in grober Nähe elektrischer Ladungen. Die Thiersuchungen Rutherfords über den Zusammenstob von $\alpha$-Teilchen mit Atomen leichter Elemente, insbesondere mit Wasserstoff (Phil. Mag. 37, S. 357, 1919) haben ergeben, daß sich die $\alpha$-Partikel wie zweifach positiv geladene Scheibahen von einem Halbmesser von höchsteas $3 \cdot 10^{-13} \mathrm{~cm}$ verhalten, die sich in der Richtung ihrer Symmetrieachso stets paraliel zu sich selbst fortbewegen. Diesen Anforderungen genügt das Lenzsche Heliumkernmodell (Münchn. Ber. 1918, S. 355) ausgezeichnet, wonach ein $\alpha$-Teilchen aus einem von vier Wasserstoffkernen gebildeten "Ring“ bestehen soll, der symmetrisch zu zwei auf der Achse desselben befindlichen, bezüglich des Madellschwerpunktes ruhenden Elektronen rotiert. Rechnet man dieses Modell mit Coulombschen Kräften und mit der Quantenthecrie durch, so erhält man für dasselbe einen viel zu geringen Energieinhalt im Vergleich zu dem nach der speziellen Relativitätstheorie berechneten Energieinhalt und der dementsprechend auch von Rutherford gefundenen hohen Stabilität der $\alpha$-Teilehen. Bringt man hingegen an Stelle des Coulombschen Gesetzes e/r das allgemeine Kraftgesetz e/rn in Ansatz, so kann man n. mit Hilfe des bekannten relativistischen Energieinhalts bestimmen. Für eine mittlere Distanz von etwa $1,8 \cdot 10^{-13} \mathrm{~cm}$ findet man $n=2,11 \%$. Der Radius des H-Kernringes im $\alpha$-Teilchen betrïgt dann etwa 1,5. $10-13 \mathrm{~cm}$, die Distanz der beiden Elektrouen von der Ringebene $7 \cdot 10-14 \mathrm{~cm}$.

Inidem man nun nach diesem Modell die rüumliche Verteilung der durch $\alpha-$ Strahl-Sto $\beta$ erzeugten schnellen H-Strahlen berechnet und mit den Ergebnissen der Rutherfordschen Zühlungen vergleicht, wir'l man den Verlauf der an die Stelle des Coulombschen Gesetzes tretenden Beziehung auch für größere Distanzen als $1,8 \cdot 10-13 \mathrm{~cm}$ näherungsweise verfolgen können. I!n Gebiete der Röntgenspektren ( $K$-Serie) gilt das Colllomibsche Gesetz bereits ohne merkliche Abricichungen.

Denkt man sich beispieirweise ein $\alpha$-Teilchen und einen Wasserstoffkern - beide der Einfachheit balber als punktförmig angenommen - in einer Entfernung von $1,8 \cdot 10^{-13} \mathrm{~cm}$, so müBten sie, falls eine so enge Annäherung überliaupt möglich ist, eine abstoßende Kraft von rund $446 \mathrm{~kg}$ aufeinander ausüben, während das Coulombsche Gesetz hierfür „nur" $14 \mathrm{~kg}$ ergeben würde.

Das wichtigste an diesen Resultaten besteht wohl darin, daß die modernen Theorien daran eine Kontrolle finden können, wenn sie einmal so weit. gediehen sein werden, ïber die Verzerrung des Coulombschen Feldes in großer Nähé der elementaren Ladungen bestimmte Aussagen 'machen zu können. A. Smekal.

Metalluntersuchungen mittels Röntgenstrahlen. (S. Nishikawa und S. Asahara, Physical Review 15, 38, 1920.) Die photographische Beobachtung des beim Durchgang der Röntgenstrahlen durch eine dünne - Metallschicht auftretenden Beugungseffektes wird von den Verfassern benützt, um den Einflub mechanischer und thermischer Einwirkungen auf die Metallstruktur zu untersuchen. Als Strahlungsquelle diente eine Coolidgeröhre (Maximalspannung 60000 Volt). Die Versuchsanordnung ist eine ganz ähnliche wie die zur Aufnahme der Lauephotogramme bei Kristallen; bei $0,1^{\prime} \mathrm{mm}$ Dicke der Metallschichten betrug dir Expositionsdauer etwa 1 Stunde bei 5 Milliampere Belastung.

Zuerst wurde der Einflub des Walzens bei Silber und Zinn untersucht. Waihrend unmittelbar nach dem
Walzen ein Bild erbalten wird ähnlich dem bei der Durchstrablung amorpher Stoffe (z. B. Paraffin), ergeben sich im Laufe der nächsten Tage und Wochen Bilder, bei denen die konzentrischen Beugungsringe sich immer mehr und mehr in einzelne Flecken auflösen, so daB die letzten Bilder der Serie das typische Aussehen der Kristallphotogramme zeigen. Durch Glüben kann dieser Effekt der „Erholung von der Walzwirkung" wesentlich beschleunigt werden. Die erforderliche Glühtemperatur ist bei den verschiedenen Metallen versehieden. (30 Minuten bei einer Temperatur von 80 Grad genügen, um beim Silber den Walzeffekt rückgängig zu machen, während beim Kupfer ein zweistündiges Ausglüben bei $800 \mathrm{Grad}$ noch nicht ausreicht.)

Die starke Veränderlichkeit der Beugungsbilder bei der Annäherung an die Umwandlungstemperatur (Ubergang in eine andere Modifikation) ermöglicht eine sehr genaue experimentelle Bestimmung des Um: wandilungspunktes. Für Thallium ergibt sich als Umwandlungstemperatur 227 Grad. Dagegen konnte für $\mathrm{Zinn}$, das bei 160 Grad einen Umwandiungspunkt besitzen soll, kein solcher nachgewiesen werden.

Der Arbeit, welche für die Technik wichtige praktische Anwendungen erwarten läBt, sind eine grobe Zahl von Aufnahmen an Silber, Zinn, Cadmium, Kupfer, Thallium beigefügt.

Aus der Sitzung der American Physical Society vom 28. 2. 20 ist besonders hervorzuheben:

Die K-Serie der Röntgenstrahlen. ( $W$. Duane und W. Stenström, Physical Review 15, 328, 1920.) Die Arbeit enthält Prüzisionsmessungen des Röntgenspektrums des Wolframs in Emission und Absorption. Die Beobachtung der Spektren höherer Ordnung gestattet eine Bestimmung ider Wellenlängen der Spek trallinien (ansgedrückt in Angström) mit einer, Genauigkeit von drei Einheiten der fünften Stelle nach dem Komma. Es ist daher von großem Interesse, daß die von der Sommerfeldschen Theorie geforderte Gleichheit der Schwingungsdifferenzen des $\alpha$-Dubletts der $K$-Serie und des $L$-Serien-Dubletts durch diese Prïzisionsmessungen bestïtigt wird. Die Verfasser zeigen ferner, dab ibre Messungen in guter Uberein stimmung sind mit dem von Duane und Shimizu aufgestellten Gesetz, daß die Differenz der Frequenzen der Absorptionsbandkanten der $K$ - und $L$-Serie die Frequenz der $\alpha$-Linie der $\boldsymbol{K}$-Serie liefert. Es scheint den Verfassern nicht bekannt zu sein, daß dieses Gesetz schon im Jahre 1916 von Kossel ausgesprochen worden ist.

Die M-Serie der Hochfrequenzspektra. $(J, C$. Karcher, Physical Review 15, 285, 1920.) Zur photographischen Aufnahme der $\boldsymbol{M}$-Serie des Röntgenspektrums der Metalle Bi bis Pb wird ein Vakuumspektrograph verwandt, weloher sich von den bekannten Konstruktionen dadurch unterscheidet, daB die Strahlungsquelle innerhalb des Spektrometers angebracht ist. Gegenüber den bisher bekannten $\boldsymbol{l}$-Linien werden drei weitere, sehr schwache Linien entdeckt.

Glocker.

\section{Geophysikalische Mitteilungen.}

Die Chandlersche und die Newcombsche Periode der Polbewegung $(B$. Wanach, Zentralbureau der internationalen Erdmessung, Neue Folge der Veröffentl. Nr. 34, Berhin 1919, G. Stankiewicz). Seit Newcomb auf theoretischem Wege gezeigt hat, daB die Elastizität des Erdkörpers die Eulersche Periode der freien Schwingung, welche für die feste Erde 304 Tage betrïgt, vergrößert, zweifelt man nicht mehr daran, daß 